\title{
LOFAR: Origins and Hopes
}

\section{George Miley ${ }^{1}$}

Sterrewacht, Leiden University

Leiden, The Nederlands

E-mail: mileyestrw. Ieidenuniv.nI

I discuss how LOFAR developed from an idea into a funded project. Special attention is given to the scientific rationale for LOFAR in the Netherlands and the reasons that led to its funding. I conclude by drawing some lessons for SKA.

ISKAF2010 Science Meeting - ISKAF2010

Assen, the Netherlands

June 10-14 201

1 Speaker 


\section{Introduction}

A highlight of ISKAF2010 will be the official inauguration of the SKA path-finder, LOFAR. It is therefore fitting to begin this ISKAF week by recalling how the LOFAR project evolved from an idea to a funded project. In attempting to do this, I shall draw some lessons that can hopefully help in our quest to secure SKA funding.

My review will concentrate on the early years, from the conception of LOFAR in 1997 until the project was funded by the Dutch Cabinet in 2003. I shall give this talk from a highly personal perspective, describing the main astronomical drivers that drove the development of LOFAR within the Netherlands and the main factors that contributed to the decision by the Dutch government to fund it. I apologise for the self indulgence.

In compiling this story I have been helped by the recollections of several colleagues, who I thank at the end.

\section{Prologue: Emergence of Dutch radio astronomy and ASTRON}

I first stress some unique aspects of Dutch radio astronomy. Unlike most other countries, where radio astronomy arose through the curiosity and technical prowess of electrical engineers and happened despite astronomers, radio astronomy in the Netherlands developed out of astronomy. It was the foresight and broad astronomical vision of Jan Oort that created Dutch radio astronomy, the construction of Dwingeloo and Westerbork and the setting up of ASTRON. The SRZM, that later became the NFRA/ASTRON, was a daughter of Oort and the Dutch university astronomers, with its headquarters housed at Leiden Observatory until the early seventies.

The fact that Dutch radio astronomy was born out of astronomy rather than engineering had advantages and disadvantages. On the one hand its advancement was driven by scientific curiosity (e.g. the search for the HI line followed the prediction of its detectability by van der Hulst). On the other hand, although solid and robust, Dutch radio astronomical facilities and instruments were not among the most innovative. The development of the newest radio astronomical technology generally took place outside the Netherlands, in countries such as the US, the UK and Australia, where the engineering tradition dominated the field.

During the late eighties and early nineties the balance between science and technology in Dutch radio astronomy was reversed. ASTRON began to pioneer exciting and important cutting-edge technological developments such as phased arrays.

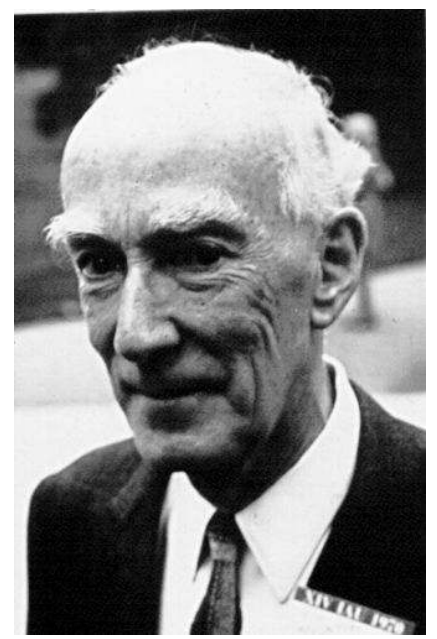

Jan Oort, father of Dutch radio astronomy

However, these were motivated more by engineering considerations and the very distant future rather than by a goal of facilitating science on foreseeable timescales. 


\subsection{Dutch radio astronomy in 1995}

Let us consider the ASTRON of fifteen years ago. In 1995, despite a recent upgrade, Westerbork was 25 years old. ASTRON was focusing much of its activities on the development of technology for a virtual facility SKA (then SKAI), that was believed to be $10-15$ years away. Some of us regarded 25 years as a more realistic estimate.

Meanwhile at the Dutch universities interest in radio astronomy was dwindling and was being supplanted by opportunities available in other regions of the spectrum, such as the large optical-infrared telescopes with their new generation of detectors.

The success of Dutch astronomy within the Netherlands compared with other sciences is in large measure due to the apparent unity projected to the outside world by the astronomical community. The diverging interests of ASTRON with the universities therefore posed a substantial threat to the future of Dutch astronomy as a whole. Furthermore there was a significant danger that shifting interests of Dutch astronomers would mean that when SKA was eventually built, radio astronomy in the Netherlands would be virtually extinct. SKA would be "a front-ranked hospital without any patients".

For these reasons, as Chairman of Leiden's astronomy department and a new member of the ASTRON Board, I felt that ASTRON's strategy needed to be modified and that its technology development program should be more attuned to the scientific interests of the university astronomers. But what should be done? It is increasingly difficult for a small country such as the Netherlands to find a niche in which it can excel both in technology and science. There were two possibilities:

(i) ASTRON could exploit their recent amalgamation with the Dutch optical instrumentation group at Roden and focus on developing the next-generation optical and infrared instruments. Participation in such activities as adaptive optics, optical interferometry (VLTI) and innovative new concepts such as Holland Ford's Polar Stratosphere Telescope (POST) [1] would offer exciting scientific possibilities that would mesh well with the technological tradition and expertise of the Dutch instrumentation efforts.

(ii) The second possibility was that ASTRON and the universities would together embark on a new radio astronomical project. Such a project would need to be realisable on a medium timescale and ideally have the potential of producing paradigm-changing science across a wide range of astronomy. It should also be inexpensive enough for the Netherlands to be a major player. Low frequencies were an obvious region where such a project might be contemplated.

\section{Digression: Low frequencies and long baselines - 1965}

Although radio astronomy was born at low frequencies, the region of the spectrum below $100 \mathrm{MHz}$ had been a highly neglected one. The problem with previous low frequency arrays, such as Bill Erickson's pioneering Clark Lake facility, had been their relatively poor angular resolution and the resultant high confusion limits. Also, ionospheric perturbations and RFI were difficult to excise from the data.

During the sixties I was privileged to be a PhD student at Jodrell Bank, working in Henry Palmer's "long baseline" group. This was before the time of tape recording interferometry and 
the Jodrell radio-link interferometer was the only show in town for mapping radio sources with arc-second resolutions.

Palmer together with his two brilliant colleagues Barry Rowson and Bryan Anderson was then pushing the baselines of interferometers out beyond $100 \mathrm{~km}$, thereby obtaining unprecedented resolution on newly discovered quasars and other discrete radio sources at frequencies of $408 \mathrm{MHz}$ and above. During 1965 Bruce Slee spent a sabbatical working within our group. As one of the pioneers of low frequency radio astronomy, it was no surprise that Bruce decided to try do a long baseline experiment at low frequencies. But would ionospheric irregularities permit fringes to be obtained over a baseline of $120 \mathrm{~km}$ at $38 \mathrm{MHz}$ ? Another postgraduate student, Peter Wraith was assigned to help Bruce with the analysis, but all our experiments were group efforts. I well remember manning the interferometer during the first night of the Slee-Wraith experiment and my excitement when I saw strong but irregular fringes appear on the chart recorder from the Crab and 3C273. This experiment showed that although low frequency interferometry over $100 \mathrm{~km}$ baselines was difficult, it could be done.

\section{The LOFAR proposal and its rationale - 1997}

Now I shall fast-forward to the Oort workshop held on 2 - 3 June 1997 at Leiden in honour of the then Oort Professor, Ron Ekers. As the organiser, I had to think of something to talk about. I had long been conscious about the neglect of low frequency radio astronomy and thought again about the Slee-Wraith experiment at Jodrell. I therefore made a plea that we should build low-frequency receivers for the EVN and conduct a VLBI campaign at frequencies below $50 \mathrm{MHz}$. Ron and I brainstormed about this at lunch and, despite the absence of alcohol, we became more ambitious. We discussed the feasibility of a building a dedicated low frequency array stretching over hundreds of kilometres.

This could be the project to fill the hole in ASTRON's strategy. A few days later, on 13 June, I sent an email to Harvey Butcher suggesting that ASTRON consider constructing a low frequency array. In July I wrote an 8-page document proposing a design study of LOFAR.

The study would last 12 months and investigate the feasibility of a survey-type array. It would have baselines of out to a few hundred $\mathrm{km}$ and operate at frequencies below $50 \mathrm{MHz}$. LOFAR would have a resolution $<5$ ' and reach flux levels $<10 \mathrm{mJy}$. I suggested that the facility be developed as a joint venture between ASTRON and NOVA (the new federated astronomy research school of the Dutch universities). This original concept was for a much less ambitious facility than the high-tech array that LOFAR eventually became. I estimated crudely that such an array might be built for 20M Dfl (about 12M€ in 2010) and might even be funded through the existing ASTRON-SKA development budget. Although this was before EoR became such a hot topic, the proposed design study would also investigate the feasibility of extending the frequency of such an array up to $150 \mathrm{MHz}$ to study $\mathrm{HI}$ in the early Universe.

A convincing strategic case could be made for such an array.

- LOFAR would be scientifically useful. It could cater to a broad range of scientific interests and would open up one of the few hitherto unexplored spectral windows.

- LOFAR would be technologically useful as a test bed for SKA technology such as phased arrays and RFI excision. 
- LOFAR would be politically useful. It would keep radio astronomy alive at the Dutch universities, function as a bridge between ASTRON and the universities and provide a natural niche for each university. Most important, a new radio telescope within the Netherlands would provide a highly visible presence for astronomy within the country. The scientific case for LOFAR was broad with the following highlights:

- Exploring new parameter space. The most important science driver that motivated the LOFAR proposal was the prospect of exploring new parameter space. I hoped and still hope that the low energy extreme of the electromagnetic spectrum will reveal new classes of exotic object and/ or new phenomena, as was the case with previous unexplored spectral windows. For frequencies below $50 \mathrm{MHz}$ the prospects for such unexpected discoveries are high, because it is a realm where strange exotic radiation mechanisms take over from synchrotron emission and the Sun and Jupiter become the brightest objects in the sky.

We all know that serendipity is an insufficient driver to sell a new facility to the powers that be. More was needed. There was and is a great deal more. Surveying at low frequencies preferentially picks up objects with steep radio spectra. This pointed to two obvious key drivers that are both dear to my heart.

- Formation of the most massive galaxies and clusters. Luminous ultra-steep-spectrum radio sources tend to be located at high redshifts $(z>2)$ and associated with the most massive and spectacular galaxies in the early Universe. These have the properties expected of progenitor brightest cluster galaxies surrounded by galaxy overdensities and pinpoint the first protoclusters [2]. Surveys at low radio frequencies are sensitive to the steepest spectra and ultra-steep spectrum radio sources tend to be at the highest high redshift. Surveys with LOFAR can therefore probe the formation of the massive galaxies, protoclusters and massive black holes in the early Universe. Perhaps most exciting, redshifted HI absorption in $\mathrm{z}>6$ LOFAR sources would be a unique diagnostic of the Epoch of Reionization and a complement to HI emission studies.

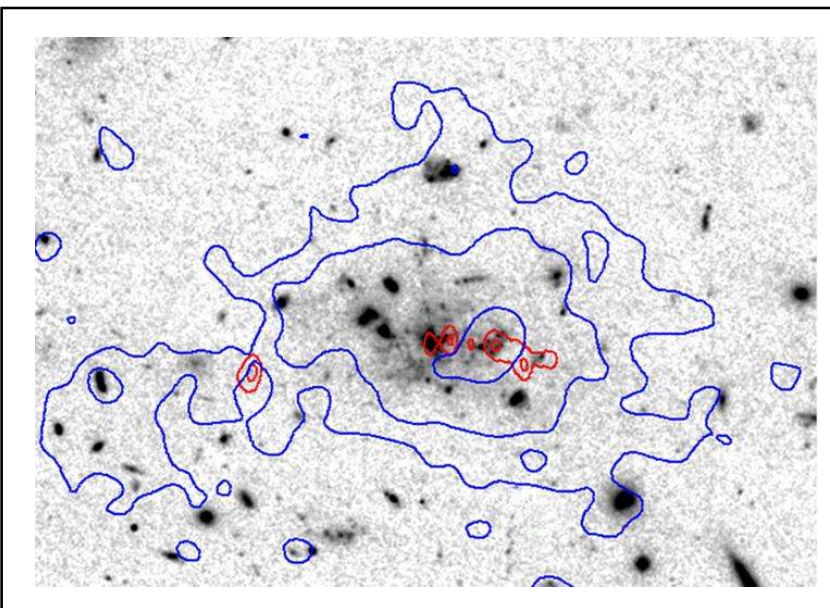

Deep Hubble image of the Spiderweb radio galaxy at the centre of a protocluster at $\mathrm{z}=$ 2.2, showing VLT Ly $\alpha$ contours (blue) and VLA $8 \mathrm{GHz}$ radio contours (red) superimposed on the composite ACS image (see [2]. The Ly $\alpha$ gaseous nebula ( 200 $\mathrm{kpc})$ is comparable in size with local $\mathrm{cD}$ galaxies.

Hosts of HzRGs are unique laboratories for studying processes in massive galaxy The LOFAR surveys should facilitate such studies to $\mathrm{z}>6$

Nature and evolution of magnetic fields in clusters. The spectra of several classes of radio sources in clusters tend to be ultra-steep [3]. These include halo sources and tailed sources. Both classes of sources probe the cluster magnetic fields and their interaction with 
the X-ray hot gas. Tailed sources are tree-rings for tracing the motion of galaxies in clusters [4]. LOFAR can push studies of diffuse cluster radio sources out to higher redshifts and sample faint sources in superclusters.

In addition to these three key drivers, the proposal mentioned a broad range of topics for which a low-frequency array would produce new science. For example, probing the spectral cutoff of radio sources associated with galaxy nuclei below $50 \mathrm{MHz}$ and mapping this with sufficient resolution would be a unique new diagnostic tool for probing gas in galaxies.

\section{Dutch LOFAR 1998 - 2002}

At the beginning of 1998, Harvey Butcher the then Director of Astron, asked Jaap Bregman one of its brightest engineers, to look into the feasibility of an array based on the LOFAR proposal. Jaap carried out a 6-month study and as a result, ASTRON pushed by Arnold van Ardenne begun a development programme to realize LOFAR.

Simultaneously with the technical developments, a group of astronomers began refining the Dutch science case for LOFAR in December 1998 under the chairmanship of Frank Israel, with Michiel van Haarlem as Secretary. This group included Robert Braun, Frank Briggs, Ger De Bruyn, Rob Fender, Huub Röttgering, Ben Stappers and Frank Verbunt. They produced a document outlining a comprehensive Dutch science case for LOFAR in January 2000.

In December 2000 LOFAR became an international project (see Section 6) and the "Netherlands LOFAR Steering Group" was set up, which I chaired. This was an oversight committee that monitored the progress of LOFAR from the perspective of Dutch astronomers. It consisted of representatives from ASTRON, the interested universities (Amsterdam, Groningen, Leiden, and Nijmegen) and the federated university research school, NOVA.

\subsection{The Dutch key science projects}

Between 1999 and 2002 a set of Dutch LOFAR key projects emerged that reflected both the specializations of the different universities and the flexibility and sophistication of the emerging technical design for LOFAR. The frequency range had increased to reach beyond 200 $\mathrm{MHz}$, and a huge field would be achieved using multiple beams. The LOFAR technical project scientist, Michiel van Haarlem called several national and international meetings during 2000, where we discussed how to relate the LOFAR scientific objective to its technical requirements. The eventual result was the emergence of the four Dutch "key projects" that has driven the development of the Dutch project since its inception. I shall describe each of them briefly.

\subsubsection{Surveys}

The key project to explore the low-frequency Universe using a series of surveys was the basis of the original LOFAR proposal (See Section 4). It needs little further description. An additional important driver for the LOFAR surveys is now to use the huge available instantaneous field to study large samples of starburst galaxies at intermediate redshifts. 
The Dutch LOFAR Key Projects

\begin{tabular}{|l|l|l|}
\hline Key Project & University & PI \\
\hline Transient Sources & Amsterdam & $\begin{array}{l}\text { Ralph Wijers } \\
\text { (originally Rob Fender) }\end{array}$ \\
\hline Epoch of Reionization & Groningen & Ger De Bruyn \\
\hline High-energy cosmic rays & Nijmegen & Heino Falcke \\
\hline Surveys & Leiden & Huub Röttgering \\
\hline
\end{tabular}

\subsubsection{Epoch of Reionisation}

In 1990 Douglas Scott and Martin Rees published a paper on the diagnostics that could be obtained about emerging large scale structure in the early Universe from redshifted HI [5]. In 1997 there followed two important theoretical papers on reionisation by Madau et al and by Gnedin and Ostriker [6,7] Ger De Bruyn and Frank Briggs (then at Groningen) had long been interested in studying damped Lyman absorbers at high-z and were pushing for LOFAR to be extended to high frequencies for that purpose. In the early summer of 1998 Peter Shaver approached Ger and Frank about the feasibility of studying the EoR with redshifted HI. This led them to write a paper which gave a practical discussion of the matter [8].

The EoR became an obvious driver for LOFAR and Ger organised an informal discussion about this in December 1998. Although extending LOFAR to cover EoR frequencies meant a considerable increase in its cost, the case for including EoR was compelling. In 2001 a twofrequency system that included high-band antennas as well as low-band ones and a substantial area in a compact central array were incorporated into the design of LOFAR.

\subsubsection{Transient Sources}

Due to the large instantaneous field provided by the multibeam design, LOFAR had become an ideal telescope to search for transient sources. In late 1999, Ben Stappers and Rob Fender (then at Amsterdam) begun discussing this possibility and in $2000-2001$ the study of transients became a key project. It fitted well with the traditional interests of the Amsterdam group in high-energy astrophysics. With a combination of a large field and high time resolution, LOFAR could explore new parameter space to search for new classes of objects and study exotic phenomena such as accreting black holes, pulsars, neutron stars, extrasolar planets and the counterparts of gravitational wave sources

\subsubsection{High-energy cosmic rays}

Studying the nature of ultra-high energy cosmic rays (energies $\sim 10^{15}-10^{20.5} \mathrm{eV}$ ) became the fourth LOFAR key project. Its origin goes back to the Soviet Union of the sixties and comes to LOFAR via Dublin, Jodrell Bank and Bonn. Trevor Weekes relates [9] that in 1962 Arkaryan published a paper predicting that extensive air showers would emit coherent geosynchrotron emission at frequencies below $200 \mathrm{MHz}$ [10]. Neil Porter, a cosmic ray expert at University College Dublin, knew Russian, spotted the paper and showed it to John Jelley, his collaborator at Harwell. Jelley contacted Bernard Lovell at Jodrell Bank and in 1964 a HarwellDublin team was sent to Jodrell to search for pulses emitted by the cosmic ray showers. They constructed a device built for the purpose that operated at $\sim 44 \mathrm{MHz}$ and was triggered by an 
array of Geiger counters set off by the showers. Coincidentally this experiment occurred simultaneously at Jodrell with Bruce Slee's long baseline interferometry at $38 \mathrm{MHz}$. Jelley et al detected a pulse and published their detection in Nature [11].

This work remained almost forgotten until Peter Bierman mentioned it to Heino Falcke and Namir Kassim at Bonn in early 2001. In March Heino sent an email to Harvey Butcher suggesting that LOFAR be used to study cosmic rays and presented this suggestion at a LOFAR meeting at Dwingeloo in May. It was incorporated as a LOFAR key project and Heino went on with Peter Gorham to develop the successful pre-LOFAR Lopes experiment [e.g. 12].

\section{The first "LOFAR International" $1999-2003$}

As the interest in LOFAR increased among Dutch astronomers, an international LOFAR dimension developed. During 1999 ASTRON approached several potential partners. In September a formal collaboration was set up between ASTRO and NRL. NRL staff (e.g. Namir Kassim and Kurt Weiler) had been involved in low frequency astronomy with Bill Erickson for several years. In February 2000 MIT Haystack joined the consortium and a governing body for the project was established with Harvey Butcher (ASTRON), Phil Schwartz (NRL) and Joe Salah (MIT) members of this LOFAR International Science Consortium (ISC).

During the next 4 years the ASTRON-NRL-MIT collaboration functioned well, with an agreed division of hardware and software tasks. NRL was responsible for developing a type of low-band antenna and MIT's responsibility was for the high-band antennas and some specific calibration software, but most of the system development work was carried out in Dwingeloo. Space management techniques were introduced and early in 2002 Jan Reitsma, previously a project manager at Fokker Space, was hired to manage the Dutch LOFAR project. An international LOFAR Project Scientist (Namir Kassim) and a Technical Project Scientist (Michiel van Haarlem) were appointed. NRAO, while not an official partner participated with observer status. Weekly telecoms were held between Dwingeloo, NRL and Haystack. In September 2003 Australia joined the collaboration and Brian Boyle became the CSIRO representative on the ISC. All this activity culminated in a two-stage Preliminary Design Review held in June 2003 at Assen and October 2003 at Alexandria Virginia.

Selecting a site for LOFAR was a thorny question. Three possible sites were considered by the ISC - West Texas, Western Australia and northern Netherlands. All three sites were deemed to be acceptable. Although the RFI situation was somewhat better in Australia, it was clear that the availability of funding and politics would be an important factor in the eventual site selection

Until 2003 there was no obvious source of funding for LOFAR. The emperor had no clothes! However, a series of developments occurred in the Netherlands (see next session) that culminated on 28 November 2003 with the award of 52M€ by the Dutch Cabinet to the project. A necessary condition was that LOFAR be built in the Netherlands. Preferring to see LOFAR built in RFI-quieter Australia, NRL, MIT and CSIRO resigned from the consortium. Despite several attempts to reach a compromise (e.g. both southern and northern LOFARs), none could be found. The "first LOFAR international" had come to an end. 


\section{LOFAR becomes a sensor array $2002-2003$}

A landmark in the development of LOFAR was its transformation into a general "sensor array" with LOFAR applications in geophysics, agricultural science and ICT, besides astronomy. This broadened the constituency of LOFAR and created a new set of stakeholders. From then onwards LOFAR would look downwards as well as upwards.

The story goes that a geophysics student at the Technical University at Delft, Gerrit Toxopeus, heard about LOFAR from one of his friends, Jeremy Butcher. He told Kees Wapenaar, Professor of Applied Geophysics, an expert on using seismic and methods to investigate the earth subsurface. This resulted in an invitation to Jeremy's father, Harvey, to give a colloquium at Delft. The rest is history. Guy Drijkoningen became leader of an exciting LOFAR application to monitor changes in the Dutch subsurface at various depths and study possible effects on it due to gas mining (e.g. gas-induced earthquakes). LOFAR could now be sold as a facility that would be of direct economic benefit to society. Furthermore, interesting cross-fertilizing discussions took place between some astronomers and geophysicists about the similarities and differences of the interferometric techniques used in both applications.

Shortly after the incorporation of geophysics in LOFAR, ASTRON contacted Daan Goense and his group at Wageningen, who were interested in using sensor arrays to optimize "precision agriculture". It became clear that ASTRON's expertise in sensor arrays would add considerable value to the agricultural research. Developing wireless sensor technology as a tool for precision agriculture became another official LOFAR application. Providing farmers with precise information on the usage of pesticides and fertilizers could avoid unnecessary spraying of chemicals. The LOFAR application involved the measurement of humidity in test fields and modelling the spread of disease using computers. I was particularly pleased that the disease adopted for study was a phytophthora (potato blight). This was the culprit that ravaged my birthplace of Ireland between 1845 and 1852. The Great Famine reduced the population of Ireland by a quarter. One million Irish perished and another million emigrated.

ICT became the third application in the LOFAR portfolio and an obvious one. After all LOFAR would be a "software telescope" with a beam steered by a computer and dependant on a substantial number of computer algorithms and a huge amount of sophisticated software.

Finally, an essential ingredient for securing LOFAR funding was the involvement of industry at all levels. Through influential firms such as IBM, Dutch Space and Ordina, a powerful industrial lobby was created.

\section{Funding for LOFAR $2002-2003$}

Fortuitously ASTRON is located in Drenthe, one of the least developed provinces in the Netherlands. Just as is the case for Green Bank in West Virginia, the presence of ASTRON and radio astronomy is regarded by the regional authorities as important stimulus for the "knowledge economy" of the province. From the outset ASTRON took special care to inform the politicians and civil servants of Drenthe in LOFAR. Relus ter Beek, the influential governor of the province and ex-Minister of Defence (now deceased) was a champion of LOFAR during these critical two years. He lobbied for LOFAR throughout the corridors of power and 
persuaded the Northern provinces (Drenthe, Groningen and Friesland) to allocate 10M€ of prefunding. He played the same role that the late Senator Byrd played in raising funds for the Green Bank Telescope, without insisting that LOFAR be called the Relus ter Beek Array!

LOFAR was too large a project to be funded by the Northern provinces alone. A national source of funding was needed. A golden opportunity arose in 2003. In their wisdom, the Dutch government has set aside a portion of revenues from our gas fields to be used for stimulating the knowledge economy and every decade or so issues a call for relevant proposals. One such an announcement of opportunity was issued with a deadline in March 2003. A Dutch consortium led by ASTRON submitted a proposal to build LOFAR, a general purpose sensor array.

The next months were tense. The scientific merit of the proposals was reviewed by a committee of the Royal Netherlands Academy of Arts and Sciences (KNAW) under the chairmanship of Harry van der Laan. Their advice in May was positive, but was later balanced by a negative advice on the economic worth of LOFAR that caused the misnamed "Committee of Wise People" to advise the Dutch Cabinet against the project in September 2003. During the following months a brilliant lobbying campaign was orchestrated by Eugene De Geus, the then Deputy Director of ASTRON and someone with exceptional networking skills. Considerable attention was paid to LOFAR in the media, including coverage by the popular Dutch TV newsdocumentary programme, "Netwerk". Our great supporter was the visionary Minister of Education, Culture and Science, Maria van der Hoeven and an enthusiastic group of senior civil servants who supported her. We worked hard, doing such things as targeting letters to junior ministers who were rumoured not to favour the allocation of funds to LOFAR. Our MIT colleagues managed to even co-opt the US Ambassador to the Netherlands into our lobbying campaign.

In November 2003 the decision about LOFAR funding came up for discussion by the Dutch Cabinet. It is rumoured that at the end the only Minister that did not support it was the then Minister of Finance, Gerrit Zalm. He apparently changed his mind when he was presented with the suggestion of a creative civil servant that funding for LOFAR that the project be prefinanced from the 2010 budget. We are now in the "year of reckoning" and Gerrit Zalm is no longer Minister of Finance. (He is CEO of the largest Dutch bank). The lobbying was successful and the Dutch Cabinet decided to allocate funding of 52M€ to the development of the LOFAR infrastructure in Drenthe. Subsequently, the Northern Provinces allocated an additional $22 \mathrm{M} €$ to the project, mainly devoted the geophysics and agricultural applications of LOFAR and the development of the High Band Antennas.

After the resignation of NRL, MIT and CSIRO from the project because of their unwillingness to contribute to the construction of LOFAR in the Netherlands, LOFAR became a predominantly Dutch project. Michiel van Haarlem became LOFAR Project Scientist and Jan Reitsma LOFAR Project Manager. Although not sufficient to build as ambitious a facility as envisaged by the original international consortium and a de-scope was needed, e.g. a LOFAR with maximum baseline of $\sim 100 \mathrm{~km}$ instead of $300 \mathrm{~km}$ and a substantial reduction in collecting area. Nevertheless LOFAR became a revolutionary facility that will explore the low-frequency sky with unprecedented resolution and sensitivity.

In the years that followed, partly thanks to an impressive ambassadorial role of Heino Falcke as International LOFAR Project Scientist, the second "LOFAR" International was 
formed. Several additional European partners joined LOFAR, most notably Germany, the UK, Sweden, France, Italy and Poland. This week will see the signing of a formal agreement to initiate a new international governance model for the LOFAR International Telescope.

\section{Hopes and lessons for SKA}

I see several lessons for SKA that can be drawn from the early history of LOFAR:

- LOFAR was driven by ASTRON, an organization with a secure annual funding stream. This is also the case for ESO's ELT. It will be more difficult to secure funding for SKA, because it is not being led by an organization with stable annual funding.

- Governments usually fund large astronomical projects for political reasons rather than through scientific altruism. Just as in the case of LOFAR and Drenthe, it will be relatively easy to obtain funding for SKA from the host country (Australia or South Africa) for chauvinistic reasons. However, additional political arguments and nonastronomical applications will probably be needed to obtain SKA funding from Europe and the USA.

- The non-astronomical applications were essential for securing funding for LOFAR. Not only did these help sell LOFAR as a project of practical benefit to society, but they created several additional constituencies and stakeholders that reinforced support for the project in The Hague. In the case of SKA, applications such as capacity building and green energy are obvious non-astronomical aspects with large potential constituencies and potentially large sources of funding.

- Building relations with influential industrial partners and making them stakeholders in LOFAR was essential. Creating an industrial lobby will be even more vital for SKA.

- Flexibility and pragmatism are essential in such a project and partners must be realistic with their expectations. It will sometimes be necessary to go from A to near B via C. There were scientific purists in the LOFAR project who refused to have anything to do with "pork" and were not willing to compromise the scientific integrity of the project. If we had listened to them, LOFAR would never have been funded. Likewise, scientific purists who refuse to consider any other aspects of SKA than its science are either unrealistic or self-serving.

- It is essential to be alert for unexpected windows of funding opportunity and exploit them, if necessary tailoring the project to take account of the new constraints.

- Building an international SKA lobby at the highest level will be crucial. Influential champions who will fight for SKA must be sought and cultivated. Who will be the Relus ter Beek or the Maria van der Hoeven of SKA? The fact that next Tuesday, three Ministers will speak to the SKA Forum illustrates the intensity of the competition between the two possible host countries. The SKA site competition has assumed such a high profile that it is doubtful whether a "winner takes all" scenario for the location of SKA is still realistic.

- A large "dollop" of luck was essential to securing funding for LOFAR and this will certainly be needed for SKA. 
Some astronomers worried that LOFAR would take funding away from large optical telescopes and we also hear this for SKA. My reply is Jan Oort's retort to H.H. Plaskett on $14^{\text {th }}$ March 1955. "I have never believed that to ask government support for a given scientific enterprise would, in the long run, diminish the chances to get similar support for other scientific purposes. This is an objection that I have often had to meet at my University. But I have never seen it happen that way. On the contrary, I have a strong impression that in this field the opposite generally holds true and that the more that is asked for the more becomes available."

I thank Jaap Bregman, Ger De Bruyn, Heino Falcke, Rob Fender and Michiel van Haarlem for their input and apologise for historical errors in this highly biased account of the early history of LOFAR. I mentioned only a few of many talented scientists and engineers at ASTRON who contributed to LOFAR's early development in the Netherlands. Other crucial players include Albert-Jan Boonstra, Michiel Brentjens, Wim van Capellen, Andre Gunst, Johan Hamaker, Jan Noordam, Kjeld van der Schaaf, Marco de Vos, and Stefan Wijnholds.

\section{References}

[1] H. Ford et al., POST: a polar stratosphere telescope Proc SPIE, 2199, pp 298-314 (1995)

[2] G.K. Miley and C. De Breuck, Distant radio galaxies and their environment, Ann Astrophys. Rev $15,67(2008)$.

[3] J.E. Baldwin and P.F. Scott, Extragalactic radio sources with steep low frequency spectra. Mon. Not. Roy. Astro. Soc., 165, 259 (1973).

[4] G.K.Miley G. C. Perola, P.C. van der Kruit and H. van der Laan, H., Active Galaxies with Radio Trails in Cluster, Nature, 237,269 (1972).

[5] D. Scott and M. Rees, $21 \mathrm{~cm}$ Line at high redshift: a diagnostic for the origin of large scale structure, Mon. Not. Roy. Astro. Soc, 247, 510 (1990).

[6] P. Madau, A. Meiksin and M. Rees, 21 Centimeter Tomography of the Intergalactic Medium at High Redshift, ApJ., 475, 429 (1997)

[7] N. Gnedin and J.Ostriker, Reionization of the Universe and the early production of metals, ApJ 486, 581 (1997).

[8] P. Shaver, R. Windhorst,, P. Madau and G. De Bruyn, Can the reionization be detected as a global signature?, A\&A 345, 380 (1999)

[9] T.C. Weekes, Radio pulses from cosmic ray air showers, First international workshop on the radio detection of high energy particles. AIP Conference Proceedings, Volume 579, pp. 3-13 (2001).

[10] G.A. Askaryan, Excess Negative Charge of the Electron-Photon Shower and Coherent Radiation Originating from It. Radio Recording of Showers under the Ground and on the Moon, Soviet Physics, J.E.T.P., 14, 441 (1962)

[11] J.V. Jelley, J.F. Fruin, N.A. Porter, T.C. Weekes, F.G. Smith and R.A. Porter, Radio Pulses from Extensive Cosmic-Ray Air Showers, Nature 205, 327 (1965)

[12] H. Falcke et al. Detection and imaging of atmospheric radio flashes from cosmic ray air showers, Nature, 435, 313 (2005). 